\title{
COMPARATIVA ENTRE LA AUTOEVALUACIÓN DEL ALUMNO Y LA EVALUACIÓN DEL PROFESOR EMPLEANDO E-RÚBRICAS EN EL PRACTICUM
}

Comparative between students' self-assessment and teacher evaluation using e-rubrics in Practicum

Comparação entre auto-avaliação ea avaliação do professor utilizando e-rubricas no prática de ensino nas escolas

Derivado de una investigación apoyada por el Plan Nacional de I+D+i de Excelencia (2015-2017) [ $\mathrm{n}^{\circ}$ EDU2013-41974-P]

\section{Mario Grande de Prado (1)}

Ana Rosa Arias Gago (2)

José Luis Vázquez Fernández (3)

(1) Universidad de León, España. Teléfono: +34 987291033. Correo electrónico: mgrap@,unileon.es

(2) Universidad de León, España. Teléfono: +34 987293118. Correo electrónico: ana.arias@unileon.es

(3) Universidad de León, España. Teléfono: +34 987291000. Correo electrónico: jvazf@unileon.es

\section{Resumen}

En esta investigación comparamos las calificaciones otorgadas por los tutores académicos del Prácticum I del Grado en Educación Infantil (Universidad de León) y las obtenidas a través de la autoevaluación de los propios estudiantes mediante el uso de e-rúbricas. El trabajo deriva del proyecto de $\mathrm{I}+\mathrm{D}+\mathrm{i}$ "Estudio del impacto de las erubricas federada en la evaluación de las competencias en el Prácticum", apoyado por el Plan Nacional de I+D+i de Excelencia (2015-2017) [nº EDU2013-41974-P]. Tras el análisis realizado, en el que no se observa correlación estadística entre las calificaciones de los tutores y las de los propios alumnos, podemos considerar que el alumnado mantiene una tendencia excesivamente optimista a la hora de autoevaluarse, a pesar de Comparativa entre la autoevaluación del alumno y la evaluación del profesor empleando e-rúbricas en el 
estar empleando herramientas de evaluación, como la e-rúbrica en la que aparecen ejemplos y criterios claros de calificación, que deberían ser compartidos por ambos agentes evaluadores.

Palabras claves: Autoevaluación; Prácticum; eRúbricas; Evaluación

\begin{abstract}
In this research, we compared the qualifications of the academic tutors of Prácticum I of the Degree in Early Childhood Education (University of León) and those made as selfevaluation by the students themselves through the use of e-rubrics. This work derives from the $\mathrm{I}+\mathrm{D}+\mathrm{i}$ project "Study of the impact of federated e-rubrics in the evaluation of the competences in the Practicum". After the statistical analysis, in which there is no statistical correlation between qualifications, we can consider that the students maintain an excessively optimistic tendency in self-evaluating, even using evaluation tools, such as e-rubric with clear examples and criteria of qualification.
\end{abstract}

Keywords: Self-assessment; Practicum; eRubrics; Evaluation

\title{
Resumo
}

Neste estudo, foram comparadas as qualificações dos tutores acadêmicos do Prática de ensino I do Licenciatura em Educação Infantil (Universidade de León) com autoavaliações dos próprios alunos utilizando e-rúbricas. O trabalho vem de um projeto de R\&D "Estudo do impacto de e-rúbricas federadas na avaliação de competencias no prática de ensino nas escolas". Após a realização de análises estatísticas, onde nenhuma correlação estadística é observada entre algumas qualificações e outras, podemos considerar que o aluno mantém uma tendência excessivamente otimista avaliando-se, mesmo quando usa ferramentas de avaliação, tais como e-rúbrica em que existem exemplos e criterios claros para a avaliação.

Palavras-chave: Auto-avaliação; Prática de ensino; eRúbricas; Avaliação

\section{Introducción}

En esta investigación tratamos de indagar en las diferencias apreciadas en las calificaciones del informe de prácticas otorgadas por los tutores académicos del Prácticum I del Grado en Educación Infantil (Universidad de León), haciendo uso de Comparativa entre la autoevaluación del alumno y la evaluación del profesor empleando e-rúbricas en el 
una rúbrica, y las realizadas como autoevaluación por los propios estudiantes mediante el uso de e-rúbricas. El trabajo deriva del proyecto de $\mathrm{I}+\mathrm{D}+\mathrm{i}$ "Estudio del impacto de las erúbricas federada en la evaluación de las competencias en el Prácticum"1 puesto en marcha en el año 2015.

A modo de marco teórico, podemos indicar como el Espacio Europeo de Educación Superior (EEES) demanda un cambio sustancial en el modo de evaluar. En relación al Prácticum de Educación, como señalan Cebrián et al. (2015), este cambio supone la necesidad de analizar y optimizar los contextos de aprendizaje entre la universidad y las instituciones facilitadoras de prácticas externas. A ello se une el auge (Cabero \& Barroso, 2013) de las prácticas sustentadas en el uso de las Tecnologías de la Información y la Comunicación (en adelante TIC) y la consiguiente aplicación de metodologías e instrumentos de evaluación innovadores (Bartolomé, Martínez \& Tellado, 2014). En esta línea de integración de las TIC en los procesos de enseñanza aprendizaje surgen las eRúbricas, entendidas como herramientas para facilitar la evaluación y su transparencia.

Sin embargo la evaluación mediante rúbricas y eRúbricas es distinta según sea entendida por los tutores académicos o por los estudiantes (Brown \& Glaser, 2003; Watts, García \& Martínez, 2006). En este trabajo, profundizaremos en la formulación de juicios según profesionales y estudiantes y sus diferencias.

\section{Método}

En el estudio se consideran dos variables: evaluación por el profesor del informe de Prácticum presentado, a través de una rúbrica, y la autoevaluación del alumno mediante el uso de una eRubrica. El análisis estadístico se realiza mediante el programa SPSS.

El número total de alumnos de Practicum I del año académico 2016-17 es de 77, si bien 12 no han enviado la autoevaluación, concretando la muestra en 65 alumnos.

Tras la recopilación de datos se procede al análisis estadístico e interpretación de resultados. Con objeto de comparar la autoevaluación y la evaluación realizada por el profesor, se utiliza la prueba t para muestras relacionadas, calculando las diferencias entre los valores de las dos variables para cada alumno y contrastando si su media

\footnotetext{
${ }^{1}$ Financiado por el Plan Nacional de I+D+i de Excelencia (2015-2017) [no EDU2013-41974-P]

Comparativa entre la autoevaluación del alumno y la evaluación del profesor empleando e-rúbricas en el Prácticum 
difiere significativamente de cero para un nivel de significación $\alpha=0$ ’05 (McMillan \& Schumacher, 2005).

\section{Resultados y discusión}

a) Correlación

No encontramos correlación ( $r$ de Pearson) entre las notas de la autoevaluación y la evaluación del profesor, aun partiendo ambos de la misma herramienta (ver tabla 1).

Tabla 1.

Correlaciones

\begin{tabular}{llll}
\hline & & Calificación informe & Autocalificación \\
\hline Calificación Informe & Correlación de Pearson & 1 &,- 143 \\
& Sig. (bilateral) & &, 255 \\
& N & 72 & 65 \\
\multirow{4}{*}{ Autocalificación } & Correlación de Pearson &,- 143 & 1 \\
& Sig. (bilateral) &, 255 & \\
& $\mathrm{~N}$ & 65 & 65 \\
\hline
\end{tabular}

b) Comparación de medias

Como podemos observar la media de los tutores es inferior en prácticamente medio punto a la obtenida de los valores de la autoevaluación. Esta última muestra además una menor dispersión. (Ver tabla 2).

Tabla 2.

Estadísticos de muestras relacionadas

\begin{tabular}{lcccc}
\hline & Media & N & Desviación típ. & Error típ. de la media \\
\hline Calificación Informe & 7,9054 & 65 & 1,33062 &, 16504 \\
Autocalificación & 8,4446 & 65 &, 94489 &, 11720 \\
\hline
\end{tabular}

En la tabla 3 aparece la comparación entre las medias empleando la prueba $t$.

Tabla 3.

Prueba de muestras relacionadas

\begin{tabular}{|c|c|c|c|c|c|c|c|c|}
\hline & \multicolumn{5}{|c|}{ Diferencias relacionadas } & \multirow[t]{3}{*}{$\mathbf{t}$} & \multirow[t]{3}{*}{ gl } & \multirow[t]{3}{*}{$\mathbf{p}$} \\
\hline & \multirow[t]{2}{*}{ Media } & \multirow[t]{2}{*}{$\begin{array}{l}\text { Desv. } \\
\text { típ. }\end{array}$} & \multirow{2}{*}{$\begin{array}{c}\text { Error } \\
\text { típ. de } \\
\text { la } \\
\text { media }\end{array}$} & \multicolumn{2}{|c|}{$\begin{array}{l}\text { 95\% Intervalo de } \\
\text { confianza para la } \\
\text { diferencia }\end{array}$} & & & \\
\hline & & & & Inferior & Superior & & & \\
\hline $\begin{array}{l}\text { Calificación } \\
\text { Informe - } \\
\text { Autocalificación }\end{array}$ &,- 53923 & 1,73875 & ,21567 &,- 97007 &,- 10839 & $-2,5$ & 64 &, 015 \\
\hline
\end{tabular}

Comparativa entre la autoevaluación del alumno y la evaluación del profesor empleando e-rúbricas en el Prácticum 
Estas diferencias entre las medias, resultan estadísticamente significativas al situarse el valor de $p$ por debajo de 0,05 (McMillan y Schumacher, 2005).

\section{Conclusiones}

Tras identificar diferencias en la evaluación del tutor y la autoevaluación del informe de prácticum por parte del alumno, hemos iniciado un proceso de optimización de la rúbrica y eRúbrica de evaluación, con el fin de generar un nuevo espacio y proceso de revisión para seguir avanzando en la mejora del aprendizaje.

Nuestro próximo objetivo por tanto sería conseguir un instrumento más claro y transparente que acerque las puntuaciones de tutores y alumnos, empleando las TIC (Cebrián et al., 2015).

Con los datos obtenidos, podemos considerar que el alumnado mantiene una tendencia más positiva que sus evaluadores a la hora de calificar sus propios trabajos, incluso cuando tiene acceso a la herramienta que se empleará en la evaluación, en este caso una eRúbrica en la que aparecen ejemplos y criterios claros de calificación.

\section{Referencias}

Bartolomé, A., Martínez-Figueira, E., \& Tellado-González, F. (2014). La evaluación del aprendizaje en red mediante blogs y rúbricas: ¿complementos o suplementos? REDU. Revista de Docencia Universitaria, 12(1), 159-176.

Brown, S., \& Glaser, A. (2003). Evaluar en la universidad. Problemas y nuevos enfoques. Madrid: Nancea.

Cabero Almenara, J., \& Barroso Osuna, J. (2013). La escuela en la sociedad de la información. La escuela 2.0. En J. Cabero Almenara, y J. Barroso Osuna, Nuevos escenarios digitales (págs. 21-36). Madrid: Pirámide.

Cebrián, M. et al. (2015). Las eRúbricas para la tutorización y evaluación de los aprendizajes en las prácticas externas. En Raposo-Rivas, M. et al. (2015). Documentar y Evaluar la experiencia de los estudiantes en las prácticas (pp.253260). Poio 2015. Santiago de Compostela: Andavira. Recuperado a partir de http://redaberta.usc.es/poio/documentos/actas/actas_poio_2015.pdf

Mcmillan, J.H., \& Schumacher, S. (2005). Investigación educativa. Madrid: Pearson.

Comparativa entre la autoevaluación del alumno y la evaluación del profesor empleando e-rúbricas en el 
Watts, F., García-Carbonell, A., \& Martínez-Alzamora, N. (2006). Relación entre autoevaluación y evaluación por profesor. Estimación del tiempo de dedicación del estudiante. En Watts, F. y García-Carbonell, A. (eds.), La evaluación compartida: investigación multidisciplinar (pp. 109-130). Valencia: UPV.

Comparativa entre la autoevaluación del alumno y la evaluación del profesor empleando e-rúbricas en el 